\section{Use of Fourier- domain OCT to detect retinal nerve fiber layer degeneration in Parkinson's disease patients}

M Satue ${ }^{1,2}$, E Garcia-Martin ${ }^{1,2}$, I Fuertes ${ }^{1,2}$, S Otin'1 R Alarcia ${ }^{3}$, R Herrero',2, MP Bambo 1,2, LE Pablo ${ }^{1,2}$ and FJ Fernandez ${ }^{1,2}$

\begin{abstract}
Purpose To demonstrate axonal loss in the retinal nerve fiber layer (RNFL) of patients with Parkinson's disease (PD) and to evaluate the ability of Fourier-domain optical coherence tomography (OCT) to detect RNFL degeneration and retinal thinning in these patients.

Methods PD patients $(n=100)$ and healthy subjects $(n=100)$ were included in the study and underwent visual acuity, color vision, and OCT examinations using two nextgeneration Fourier-domain devices (Spectralis and Cirrus). Differences in the RNFL thicknesses were compared between patients and controls. Results RNFL thicknesses were significantly reduced in PD patients compared with healthy subjects, especially those obtained using the Spectralis OCT, in the inferotemporal quadrant $(155.6 \pm 16.5 \mu \mathrm{m}$ in healthy eyes $v s 142.1 \pm 24.9 \mu \mathrm{m}$ in patients, $P=0.040)$ and in the superotemporal quadrant (142.6 $\pm 20.9 \mu \mathrm{m}$ in healthy eyes $v s$ $132.77 \pm 18.6 \mu \mathrm{m}$ in PD patients, $P=0.046$ ). Significant differences were observed between controls and patients in relation to mean macular thickness $(P=0.031)$, foveal thickness $(P=0.030)$, and inferior outer thickness $(P=0.019)$.

Conclusion PD is associated with RNFL loss and retinal thinning, which is detectable by Fourier-domain OCT measurements.

Eye (2013) 27, 507-514; doi:10.1038/eye.2013.4; published online 22 February 2013
\end{abstract}

Keywords: Parkinson's disease; retinal nerve fiber layer; optical coherence tomography; biomarker; degeneration

\section{Introduction}

Parkinson's disease (PD) is a neurodegenerative process that leads to a selective loss of dopaminergic neurons, mainly in the basal ganglia of the brain. Clinical manifestations include movement alterations as well as nonmotor symptoms, such as dementia, depression, and autonomic dysfunction. ${ }^{1}$ Neurons and neural circuits outside the basal ganglia can be affected simultaneously or even before the substantia nigra. ${ }^{2}$ Vision is one of the non-motor systems altered in PD, especially the visual field corresponding to the fovea. ${ }^{3}$

The mammalian retina contains dopaminergic neurons within the inner retinal layer that modulate the receptive fields of ganglion cells through long-range lateral interactions in the inner plexiform layer. The ganglion cells produce color vision and contrast sensitivity. Previous studies have shown that vision, contrast sensitivity, absolute and temporal sensitivity, and color vision are at least partially regulated by dopamine, ${ }^{3}$ and it has been demonstrated that retinal cells in PD patients have altered dopaminergic synaptic activity compared with healthy controls. ${ }^{4}$ Multifocal electroretinogram changes have also been reported in PD patients. ${ }^{5}$

The loss of retinal ganglion cells can be detected using ocular imaging technologies such as optical coherence tomography (OCT), ${ }^{6}$ which provide noninvasive, rapid, objective, and reproducible measurements of the retinal nerve fiber layer (RNFL). Numerous studies have analyzed the ability of OCT to detect RNFL thickness abnormalities and changes in
${ }^{1}$ Department of Ophthalmology, Miguel Servet University Hospital, Zaragoza, Spain

${ }^{2}$ Aragones Institute of Health Sciences, Research Department, Zaragoza, Spain

${ }^{3}$ Department of Neurology, Miguel Servet University Hospital, Zaragoza, Spain

Correspondence: E Garcia-Martin, Department of Ophthalmology, Miguel Servet University Hospital, Padre Arrupe, Consultas Externas de Oftalmología, Zaragoza 50009, Spain. Tel: + 34976765558

E-mail: egmvivax@ yahoo.com

Received: 30 March 2012 Accepted in revised form: 26 December 2012 Published online: 22 February 2013 
the macular area in patients with neurodegenerative diseases, such as multiple sclerosis, in which axonal damage unrelated to inflammatory or autoimmune episodes against myelin occurs already in the early stages of the disease, and axonal neurodegeneration is directly related to permanent functional disability. ${ }^{7-10}$

Recent reports demonstrated decreased retinal thickness in different macular sectors in PD patients compared with healthy subjects, ${ }^{11,12}$ and alterations in mean RNFL peripapillary thickness and multifocal electroretinogram have also been observed using timedomain OCT. ${ }^{5}$ To our knowledge, spectral-domain OCT has only been used in four previous studies of PD patients, three of which demonstrated a reduction of the macular thickness (inner retinal layer); ${ }^{11,13,14}$ only one of these studies showed significant differences in RNFL thickness between PD patients and healthy controls. ${ }^{15}$ As these findings might have been influenced by the small sample sizes used, a larger sample would help to corroborate these findings.

Two Fourier-domain OCT devices were used in this study: the Cirrus (Carl Zeiss Meditec, Dublin, CA, USA) and the Spectralis (Heidelberg Engineering Inc., Heidelberg, Germany). The Cirrus OCT obtains images with $5 / 1000 \mathrm{~mm}$ resolution, while the Spectralis OCT uses polarized light to simultaneously analyze multiple wavelength data from the light reflection, acquiring 40000 layers per second. One of the most important differences between the Cirrus OCT and the Spectralis OCT is that the latter is combined with a confocal scanning laser ophthalmoscope, providing detailed simultaneous images and improved contrast.

The purpose of this study was to detect and measure RNFL defects and retinal thicknesses in PD patients compared with healthy controls using these two next-generation Fourier OCT devices, and to compare the ability of both OCT systems to detect alterations in the retina that are not evident on routine clinical examination.

\section{Materials and methods}

A total of 100 patients with PD and 100 sex- and agematched healthy subjects were included in the study. All procedures adhered to the tenets of the Declaration of Helsinki, and the experimental protocol was approved by the local ethics committee. All subjects provided informed consent to participate in the study.

The diagnosis of PD was based on UK Brain Bank Criteria, which included, in the first step, bradykinesia and one additional symptom, that is, rigidity, $4-6 \mathrm{~Hz}$ resting tremor, or postural instability. ${ }^{16,17}$ For the diagnosis of 'definite' PD, three or more of the following criteria were required in combination with those in step one: unilateral onset, progressive disorder, persistent asymmetry affecting the side of onset the most, excellent response (70-100\%) to levodopa, severe levodopainduced chorea, levodopa response for 5 years or more, and clinical course of 10 years or more. ${ }^{17,18}$ Despite clinical criteria, definite PD diagnosis requires postmortem confirmation, ${ }^{18}$ although the correlation between clinical signs and physiopathology is not very strong and Lewy bodies are only found in $76 \%$ of patients diagnosed with PD. ${ }^{17}$ The stage and severity of PD were determined based on the Hoehn-Yahr scale, a commonly used system for describing the progression of PD symptoms. ${ }^{19}$ This scale contains stages from 0 (no signs of disease) to 5 (requiring a wheelchair or bedridden unless assisted) to indicate the relative level of disability.

Inclusion criteria were confirmed PD diagnosis, best-corrected visual acuity (BCVA) of 20/30 or higher (using a Snellen chart) in each eye to enable performance of the protocol to be assessed, anterior chamber depth of Schaffer grades III and IV using indirect gonioscopy, and an intraocular pressure $<21 \mathrm{~mm} \mathrm{Hg}$ to rule out any RNFL decrease because of other processes such as openangle chronic glaucoma. ${ }^{20}$ Exclusion criteria were the presence of significant refractive errors ( $>5$ diopters of spherical equivalent refraction or 3 diopters of astigmatism), media opacifications, systemic conditions that could affect the visual system (such as diabetes or ischemic cardiopathy), a history of ocular trauma or concomitant ocular diseases (including a previous history of retinal pathology, glaucoma, laser therapy), or ocular pathologies affecting the cornea, lens, optic nerve, or retina (including macular alterations). Healthy controls were recruited from hospital staff and family members of healthy patients. Controls had no history of ocular or neurologic disease, and their BCVA was 20/30 or better according to the Snellen scale.

All subjects underwent a complete neuroophthalmologic examination, which included assessment of BCVA, color vision evaluation (using Ishihara's isochromatic charts), eye movement, pupillary, anterior segment and fundoscopic examinations, Goldmann applanation tonometry, and OCT examinations using the Cirrus HD OCT (Carl Zeiss Meditec) and the Spectralis OCT (Heidelberg Engineering Inc.). Funduscopic examination was based on the disc morphology (normal, general pallor, sectorial pallor, and edema) with a 78-diopter lens. Each eye was considered separately and only one eye of each subject was included randomly in the analyses. Disease duration, age at PD diagnosis, and treatment were also recorded.

\section{OCT evaluation}

OCT tests were performed to obtain measurements of the peripapillary RNFL using the Cirrus and Spectralis OCT 
devices, both of which were used in random order to prevent any effect of fatigue bias. All scans were performed by the same experienced operator. Between scan acquisitions, there was a time delay and subject position and focus were randomly disrupted, such that alignment parameters had to be newly adjusted at the start of each image acquisition. No manual correction was applied to the OCT output. An internal fixation target was used because it is reported to provide the highest reproducibility. ${ }^{10}$ The quality of the scans was assessed before the analysis, and poor quality scans were rejected. The Cirrus OCT determines the quality of images using a signal strength measurement that combines signal-to-noise ratio with the uniformity of the signal within a scan and is measured on a scale of 1 to 10 , where 1 is categorized as poor image quality and 10 as excellent image quality. Only images with a score $>7$ were evaluated in our study. The Spectralis OCT uses the blue quality bar in the image to indicate the signal strength. The quality score ranges from 0 (poor quality) to 40 (excellent quality). Only images with a score $>25$ were analyzed.

Following the recommended procedure for scan acquisition, the subject's pupil was first centered and focused in an iris viewing camera on the system's data acquisition screen, and then the system's line-scanning ophthalmoscope was used to optimize the view of the retina. The OCT scan was aligned to the proper depth, and patient fixation and system polarization were optimized to maximize the OCT signal.

The Cirrus OCT optic disc protocol generates $200 \times 200$ voxel images from 200 linear scans that are performed by 200 A-scans. This option analyzes a cube of width 6-mm around the optic nerve. In each series of scans, mean RNFL thickness, quadrant RNFL thickness (superior, inferior, temporal, and nasal), and thickness at the 12-clock hours of $30^{\circ}$ RNFL were analyzed. The hour sectors were assigned a number from position $\mathrm{C} 1$ to $\mathrm{C} 12$ in the clockwise direction for the right eye and in the counter-clockwise direction for the left eye. The Cirrus Macular Cube $200 \times 200$ protocol provides retinal

thickness values for the nine areas corresponding to the Early Treatment Diabetic Retinopathy Study (ETDRS). ${ }^{21}$ ETDRS areas include a central 1-mm circle, representing the foveal area, and inner and outer rings of diameter 3 and $6 \mathrm{~mm}$, respectively. The inner and outer rings are divided into four quadrants: superior, nasal, inferior, and temporal. Central foveal thickness was also calculated (Figure 1). The average retinal thickness and the total macular volume in the 6-mm diameter ETDRS ring were calculated based on the proportional contribution of the regional macular thicknesses. ${ }^{21}$ The mean of all points within the inner circle of 1-mm radius was defined as the central foveal subfield thickness. The intermediate and outer rings were subdivided into superior, nasal, inferior, and temporal inner and outer areas, respectively. The nine areas were analyzed using Cirrus software (version 3.0; Carl Zeiss Meditec).

The RNFL Spectralis protocol generates a map with a mean thickness and six sector thicknesses (superonasal, nasal, inferonasal, inferotemporal, temporal, and superotemporal in the clockwise direction for the right eye and counter clockwise for the left eye). The Fast macular protocol uses an internal fixation source and centers on the patient's fovea. The operator independently monitors the stability of fixation with the incorporated infrared camera, with the axial and transverse resolutions of the images being 7 and $10 \mu \mathrm{m}$, respectively. The retinal thickness map analysis protocol represents the nine subfields, as defined by the ETDRS, in a similar manner to the Cirrus OCT. ${ }^{21}$ RNFL and retinal acquisitions were obtained by the same observer using TruTrack eye-tracking technology that recognizes, locks onto, and follows the patient's retina during scanning, and automatically places follow-up scans to ensure the accurate monitoring of disease progression. Spectralis software (version 5.2; Heidelberg Engineering Inc.) was used.

\section{Statistical analysis}

All variables were recorded in a database created with the FileMaker Pro 8.5 program (FileMaker Inc., Concord, MA, USA). A cross-sectional analysis was performed: the independent variable was 'diagnosed with PD or not diagnosed with $\mathrm{PD}^{\prime}$, and dependent variables were the parameters obtained by the different diagnosis techniques included in the protocol. Age, sex, and intraocular pressure were considered modifying variables.

Statistical analysis was performed using the SPSS statistical package (version 19.0; SPSS Inc., Chicago, IL, USA). The Kolmogorov-Smirnov test was used to assess sample distribution. As the data were parametrically distributed, differences between patients and healthy controls were compared using the Student's $t$-test. Pearson's correlation analysis was used to determine the correlation coefficient $(r)$ and the statistical significance of the association $(p)$. Pearson's correlation coefficient $(r)$ can range between +1 and -1 : a value of 0 indicates that there is no association between the two variables. A value $>0$ indicates a positive association, that is, as the value of one variable increases so does the value of the other variable. A value $<0$ indicates a negative association, that is, as the value of one variable increases the value of the other variable decreases. Values of $P<0.05$ were considered to indicate statistically significant differences. 


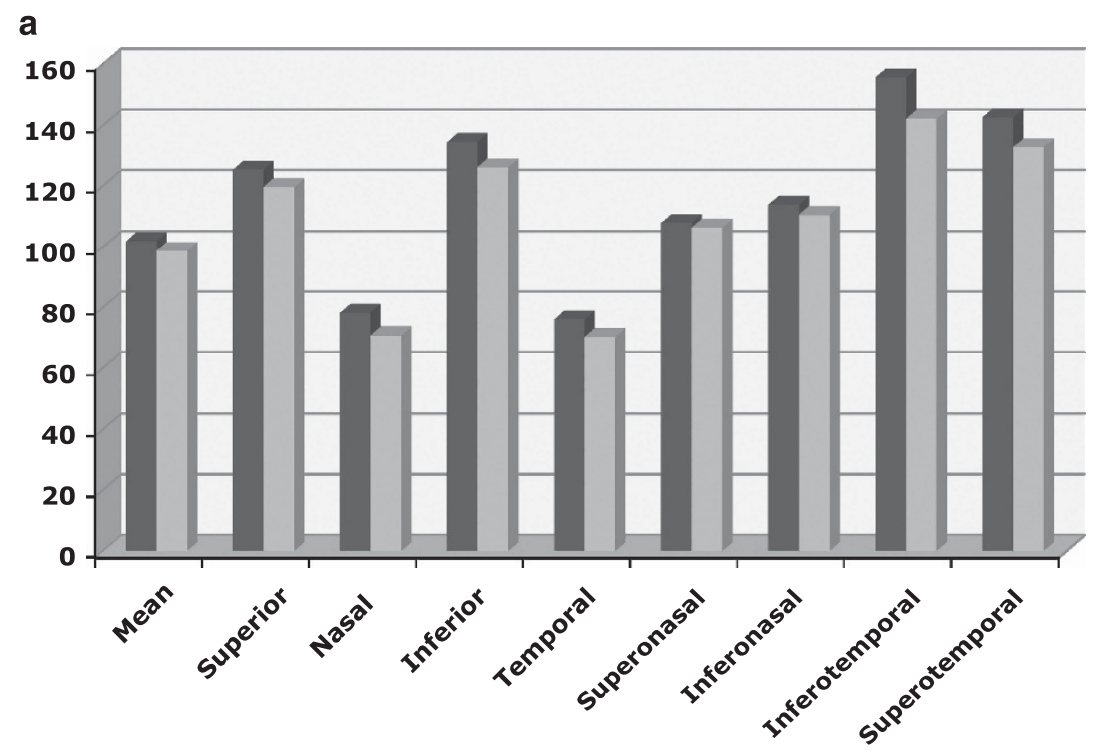

Healthy controls

Parkinson

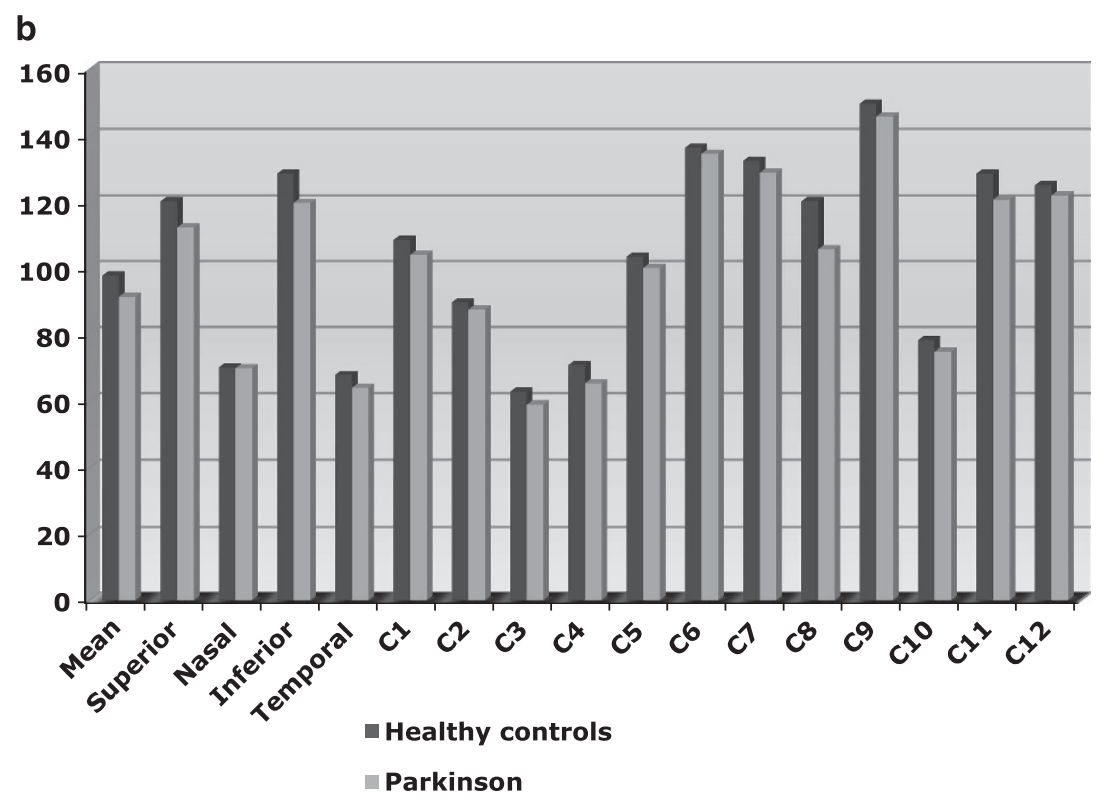

Figure 1 (a) Box plots comparing RNFL thicknesses in eyes from Parkinson disease patients and eyes from healthy subjects using Spectralis OCT. (b) Box plots comparing RNFL thicknesses in eyes from Parkinson disease patients and eyes from healthy subjects using Cirrus OCT.

Measures of each eye corresponding to the comparable parameters between both OCT devices (superior, nasal, inferior, and temporal quadrants and average RNFL thickness and the nine EDTRS macular sectors) were compared using the Student's $t$-test for paired variables.

\section{Results}

A total of 100 patients with PD and 100 healthy controls were included in the study. The mean age was 64 years (range: 54-74 years) in the PD patient group and 64 years (range: 56-72 years) in the healthy control group.

The percentage of males was $81.8 \%$ and the mean disease duration was 5.25 years (range: 2.85-7.65). Age, sex, and intraocular pressure did not differ significantly between healthy controls and PD patients $(P=0.097, P=0.154$, and $P=0.669$ respectively). The average stage of $\mathrm{PD}$ was 2.34 based on the Hoehn-Yahr scale (range: 1.50-3.00).

Received treatment was divided into three different categories: 'drugs that enhance levels of dopamine' 
(carbidopa, levodopa, and rasagiline), 'dopaminergic drugs' (pramipexole, ropirinol, and rotigotine) and 'other'(amitriptiline, propranolol, and clonazepam). 'Drugs that enhance levels of dopamine' was the most prescribed category (93\% of patients) and the combination therapy of levodopa and carbidopa the most frequent treatment $(37 \%)$. In all, $68 \%$ of treatments were categorized in 'dopaminergic', most of which were used in combination with drugs included in the previous category. A small percentage of patients (10\%) were prescribed drugs with no dopaminergic effects.

RNFL measurements evaluated in the study and the significance of differences between measurements for healthy controls and PD patients are shown in Table 1 (Spectralis measurements) and Table 2 (Cirrus measurements). The Spectralis OCT revealed significant differences in the inferior quadrants $(134.5 \mu \mathrm{m}$ in healthy controls vs $126.1 \mu \mathrm{m}$ in PD, $P=0.036)$, inferotemporal RNFL thickness (155.6 vs $142.1 \mu \mathrm{m}, P=0.040)$, and superotemporal RNFL thickness (142.6 vs $132.7 \mu \mathrm{m}$, $P=0.046)$. Other structural thicknesses showed a clear tendency to be reduced in patients with PD (Figure 1), but these differences were not statistically significant (Table 1).

Cirrus OCT measurements revealed that average RNFL thickness was significantly decreased in PD patients compared with healthy controls (inferior thickness: $128.7 \mu \mathrm{m}$ in controls $v s 120.0 \mu \mathrm{m}$ in patients, $P=0.047$; C4 thickness: 71.0 vs $65.3 \mu \mathrm{m}, P=0.024$; C8 thickness: 120.5 vs $105.8 \mu \mathrm{m}, P=0.009$; C9 thickness: 149.9 vs $146.0 \mu \mathrm{m}, P=0.037$; and C11 thickness: 128.7 vs $120.9 \mu \mathrm{m}, P=0.040$; Table 2, Figure 1).

Mean macular thicknesses were also significantly reduced in PD patients compared with healthy controls

Table 1 Mean and SD of RNFL thickness obtained using the Spectralis OCT, and comparison between healthy controls and PD subjects

\begin{tabular}{lrrr}
\hline $\begin{array}{l}\text { Spectralis OCT } \\
\text { RNFL thickness }\end{array}$ & $\begin{array}{c}\text { Healthy } \\
\text { subjects }\end{array}$ & $\begin{array}{c}\text { PD } \\
\text { patients }\end{array}$ & P-value \\
\hline Mean & $101.9(8.7)$ & $98.7(12.0)$ & 0.181 \\
Superior & $125.2(17.9)$ & $119.5(15.4)$ & 0.170 \\
Nasal & $78.9(16.5)$ & $70.8(17.5)$ & 0.145 \\
Inferior & $134.5(17.1)$ & $126.1(21.3)$ & $\mathbf{0 . 0 3 6}$ \\
Temporal & $76.1(15.3)$ & $70.3(11.9)$ & 0.099 \\
Superonasal & $108.0(24.5)$ & $106.4(21.0)$ & 0.783 \\
Inferonasal & $113.8(24.1)$ & $110.6(24.6)$ & 0.573 \\
Inferotemporal & $155.6(16.5)$ & $142.1(24.9)$ & $\mathbf{0 . 0 4 0}$ \\
Superotemporal & $142.6(20.9)$ & $132.7(18.6)$ & $\mathbf{0 . 0 4 6}$ \\
\hline
\end{tabular}

Abbreviations: OCT, optical coherence tomography; PD Parkinson disease; RNFL, retinal nerve fiber layer.

The last column shows the statistical significance of the difference between both groups. The inferior, inferotemporal, and superotemporal sectors showed significant reduction of the RNFL thickness in PD (shown in bold).
(Table 3). BCVA was also significantly different between groups (0.97 in controls vs 0.74 in PD patients, $P<0.001$ ).

Measures corresponding to the comparable parameters between both OCT devices were compared using the Student's $t$-test for paired variables as shown in Table 4.

The correlation between PD severity, disease duration, age, and OCT measurements was calculated using Pearson's correlation test. PD severity measured with the Hoehn-Yahr scale and mean disease duration (measured in years) were strongly correlated $(r=0.742, P<0.001)$, whereas only the outer superior macular thickness measured with Spectralis OCT showed a significant although mild correlation with patient's age $(r=-0.427$, $P=0.01)$.

\section{Conclusion}

The visual system is one of the non-motor systems affected by PD, and this is particularly evident with respect to the macular area. In 2004, Inzelberg et al ${ }^{22}$ demonstrated using a time-domain OCT device that RNFL thickness in the peripapillary area was reduced in a small group of 10 PD patients; these results were later confirmed by other studies using time-domain OCT. ${ }^{12,23,24}$ To our knowledge, few studies have used spectral-domain OCT ${ }^{11,13-15}$ to evaluate retinal changes in PD patients: Hajee et $a l^{11}$ and Bodis-Wollner et al ${ }^{14}$ reported a significant decrease in the inner retinal layer

Table 2 Mean and SD of RNFL thickness obtained using the Cirrus OCT, and comparison between healthy controls and PD subjects

\begin{tabular}{lccc}
\hline $\begin{array}{l}\text { Cirrus OCT } \\
\text { RNFL thickness }\end{array}$ & $\begin{array}{c}\text { Healthy } \\
\text { subjects }\end{array}$ & $\begin{array}{c}\text { PD } \\
\text { patients }\end{array}$ & P-value \\
\hline Average & $97.9(8.5)$ & $91.2(9.8)$ & 0.145 \\
Superior & $120.5(11.4)$ & $112.4(11.0)$ & 0.245 \\
Nasal & $70.2(9.9)$ & $70.0(13.8)$ & 0.567 \\
Inferior & $128.7(14.5)$ & $120.0(15.6)$ & $\mathbf{0 . 0 4 7}$ \\
Temporal & $67.8(9.0)$ & $63.9(11.9)$ & 0.237 \\
C1 & $108.7(22.2)$ & $104.2(20.9)$ & 0.224 \\
C2 & $89.6(20.1)$ & $87.5(16.8)$ & 0.505 \\
C3 & $62.7(12.5)$ & $58.9(13.0)$ & 0.081 \\
C4 & $71.0(16.4)$ & $65.3(12.6)$ & $\mathbf{0 . 0 2 4}$ \\
C5 & $103.6(24.8)$ & $100.2(24.6)$ & 0.423 \\
C6 & $136.4(29.0)$ & $134.6(29.4)$ & 0.716 \\
C7 & $132.5(24.7)$ & $129.0(24.0)$ & 0.400 \\
C8 & $120.5(27.9)$ & $105.8(12.5)$ & $\mathbf{0 . 0 0 9}$ \\
C9 & $149.9(14.5)$ & $146.0(17.3)$ & $\mathbf{0 . 0 3 7}$ \\
C10 & $78.3(16.8)$ & $74.9(12.7)$ & 0.185 \\
C11 & $128.7(22.3)$ & $120.9(21.5)$ & $\mathbf{0 . 0 4 0}$ \\
C12 & $125.2(21.9)$ & $122.2(24.3)$ & 0.453 \\
\hline
\end{tabular}

Abbreviations: OCT, optical coherence tomography; PD, Parkinson disease; RNFL, retinal nerve fiber layer.

The last column shows the statistical significance of the difference between both groups. The areas that showed a significant RNFL reduction in $\mathrm{PD}$ patients were inferior sector, and the following clock sectors: C4, C8, C9, and C11 (shown in bold). 
Table 3 Mean and SD of macular thickness parameters obtained using the Cirrus and Spectralis OCT devices, and comparison between healthy controls and PD subjects

\begin{tabular}{lccccccc}
\hline \multirow{2}{*}{ Macular thickness } & \multicolumn{3}{c}{ Cirrus OCT } & & \multicolumn{3}{c}{ Spectralis OCT } \\
\cline { 2 - 3 } & Healthy subjects & PD patients & P-value & & Healthy subjects & PD patients & P-value \\
\hline Fovea & $271.3(31.2)$ & $260.5(24.7)$ & $\mathbf{0 . 0 3 0}$ & & $263.9(20.9)$ & $256.4(27.6)$ & $\mathbf{0 . 0 3 4}$ \\
Superior inner & $326.7(17.4)$ & $322.3(19.1)$ & 0.165 & & $330.5(10.8)$ & $323.2(16.9)$ & 0.345 \\
Nasal inner & $326.7(17.4)$ & $322.3(19.2)$ & 0.136 & & $334.5(14.2)$ & $326.7(18.2)$ & 0.126 \\
Inferior inner & $322.7(14.6)$ & $319.2(18.7)$ & 0.230 & & $328.6(13.0)$ & $319.6(17.1)$ & $\mathbf{0 . 0 1 4}$ \\
Temporal inner & $315.3(16.1)$ & $310.1(18.1)$ & 0.087 & & $317.9(15.6)$ & $310.1(17.2)$ & $\mathbf{0 . 0 4 5}$ \\
Superior outer & $285.1(18.3)$ & $282.3(16.9)$ & 0.359 & & $285.1(11.2)$ & $281.8(15.8)$ & 0.098 \\
Nasal outer & $301.7(13.4)$ & $294.4(27.1)$ & 0.052 & & $301.6(13.4)$ & $297.9(29.6)$ & 0.334 \\
Inferior outer & $278.4(16.8)$ & $270.7(20.0)$ & $\mathbf{0 . 0 1 9}$ & & $274.6(11.2)$ & $274.6(12.4)$ & $\mathbf{0 . 0 5 0}$ \\
Temporal outer & $268.7(17.2)$ & $266.9(17.8)$ & 0.572 & & $269.3(29.4)$ & $268.0(14.8)$ & 0.287
\end{tabular}

Abbreviations: OCT, optical coherence tomography; PD, Parkinson disease.

The $P$-value columns show the statistical significance of the difference between both groups. Cirrus OCT showed a significant reduction in retinal thickness in the foveal area and the inferior outer sector. Spectralis OCT also showed a decrease in the inferior inner and temporal inner sectors (shown in bold).

and foveal thickness, respectively, in PD patients, but RNFL measurements were not analyzed. Aaker $e^{2} \mathrm{al}^{13}$ measured the inner retinal layer, macular thickness, and RNFL of 18 eyes in PD patients, and found significant changes in macular thickness (thinning and thickening), but no alterations in the overall average RNFL thickness, possibly because of the small sample size. Longitudinal studies with larger sample sizes were therefore suggested for these findings to be corroborated..$^{25}$

Based on the distribution of Lewy bodies at different stages of PD, Braak et $a l^{2}$ suggested that this neurodegenerative process progresses from nondopaminergic neuronal areas to central neurons in a cranio-caudal direction, and affects neurons with long axons. The RNFL is composed of ganglionic cell axons, but the presence of Lewy bodies in these cells has not yet been established by histologic studies.

Physiologic aging causes neuronal degeneration and therefore reductions in most of the regional thicknesses of the peripapillary RNFL and macula, except for the temporal quadrant of the RNFL and the corresponding 8, 9, and 10 o' clock sectors. ${ }^{26}$ In neurodegenerative diseases, the temporal quadrant is affected at an earlier stage, ${ }^{27}$ with subsequent expansion to the papillomacular sector.

Of the different digital imaging analysis techniques available, OCT is considered the most useful for the diagnosis and follow-up of optic neuropathies, and several investigators have demonstrated the capacity of OCT to evaluate neurodegenerative pathologies such as optic neuritis, ${ }^{27}$ multiple sclerosis, ${ }^{7,28}$ and Alzheimer's disease. ${ }^{29}$ In this study, two next-generation Fourierdomain OCT devices were used to evaluate RNFL and retinal thicknesses. Both devices were compared and significant differences were found between the Cirrus OCT and Spectralis OCT. These results indicate that only
Table 4 Mean and SD of difference in measurements performed using Cirrus and Spectralis optical coherence tomography devices, and statistical significance $(P)$ in both groups of patients evaluated in the study

\begin{tabular}{|c|c|c|c|c|}
\hline & $\begin{array}{c}\text { Healthy ey } \\
\text { Mean } \\
\text { difference } \\
\text { (Cirrus- } \\
\text { Spectralis } \\
\text { measurements) }\end{array}$ & P-value & $\begin{array}{l}\text { Parkinson e } \\
\text { Mean } \\
\text { difference } \\
\text { (Cirrus- } \\
\text { Spectralis } \\
\text { measurements) }\end{array}$ & P-value \\
\hline \multicolumn{5}{|l|}{ Macular parameters } \\
\hline Fovea & $-16.25(15.98)$ & $<0.001$ & $-19.24(13.68)$ & $<0.001$ \\
\hline $\begin{array}{l}\text { Superior inner } \\
\text { macula }\end{array}$ & $-15.62(6.22)$ & $<0.001$ & $-14.68(10.95)$ & $<0.001$ \\
\hline Nasal inner macula & $-14.64(8.40)$ & $<0.001$ & $-14.89(9.43)$ & $<0.001$ \\
\hline $\begin{array}{l}\text { Inferior inner } \\
\text { macula }\end{array}$ & $-15.22(6.29)$ & $<0.001$ & $-13.34(8.08)$ & $<0.001$ \\
\hline $\begin{array}{l}\text { Temporal inner } \\
\text { macula }\end{array}$ & $-14.97(8.33)$ & $<0.001$ & $-14.35(8.22)$ & $<0.001$ \\
\hline $\begin{array}{l}\text { Superior outer } \\
\text { macula }\end{array}$ & $-13.86(9.53)$ & $<0.001$ & $-10.64(6.99)$ & $<0.001$ \\
\hline Nasal outer macula & $-11.08(9.21)$ & $<0.001$ & $-12.27(7.83)$ & $<0.001$ \\
\hline $\begin{array}{l}\text { Inferior outer } \\
\text { macula }\end{array}$ & $-18.06(18.47)$ & $<0.001$ & $-12.35(9.66)$ & $<0.001$ \\
\hline $\begin{array}{l}\text { Temporal outer } \\
\text { macula }\end{array}$ & $-20.66(20.94)$ & $<0.001$ & $-12.43(10.67)$ & $<0.001$ \\
\hline \multicolumn{5}{|l|}{ RNFL parameters } \\
\hline Average thickness & $-5.89(4.23)$ & $<0.001$ & $-5.76(3.78)$ & $<0.001$ \\
\hline Superior quadrant & $-4.79(14.45)$ & $<0.001$ & $-4.50(12.42)$ & $<0.001$ \\
\hline Nasal quadrant & $-1.54(14.84)$ & $<0.001$ & $-3.06(9.34)$ & $<0.001$ \\
\hline Inferior quadrant & $-7.72(11.58)$ & $<0.001$ & $-5.93(8.24)$ & $<0.001$ \\
\hline Temporal quadrant & $-5.92(9.49)$ & $<0.001$ & $-5.42(7.16)$ & $<0.001$ \\
\hline
\end{tabular}

Significant differences were found between both devices in each group (healthy controls and Parkinson patients).

one OCT device should be selected for monitoring a patient's disease, as the measures differ. Although the reduction in RNFL thickness was not significant for some of the sites examined, the tendency for reduced RNFL thickness may have been significant for more parameters if a larger sample size had been examined.

Some recent OCT studies suggest asymmetrical involvement of the retina in PD and suggest including both eyes of each patient in the study. ${ }^{30}$ Our sample 
could have been larger and it is possible that the diagnostic yield in this study was decreased by our inclusion of a potentially less affected eye. Incorporating both eyes of a patient into the study, however, could also mask minimum symmetric structural and functional alterations. The majority of authors consider the inclusion of only one eye of each patient adequate for statistical analysis because RNFL measurements correlate significantly between the two eyes; thus, we included only one eye per patient.

Macular thickness also showed a significant reduction in the central 1-mm diameter circle, as well as for the temporal and inferior quadrants of the 3-mm ring and inferior quadrant of the 6-mm ring, in PD patients compared with controls. The inner retinal layer contains both the RNFL and dopaminergic amacrine cells, with a decrease in the retinal thickness partially because of the affectation of these cells. However, currently available OCT software is not able to determine the thicknesses of the different retinal layers, so the correlation between the dopaminergic cells and RNFL loss is still uncertain.

This study suggests that areas with reduced macular thickness also show decreased peripapillary RNFL

thickness. This could be due to the anatomic correspondence of the ganglion cells in the retina and optic nerve head. Recent studies indicate that macular thickness as a high discriminating parameter in other diseases affecting the peripapillary RNFL thickness, such as open-angle glaucoma. ${ }^{31}$

We found a significant correlation between PD severity and disease duration, and between RNFL (outer superior macular thickness) and age. These results were expected, because PD is a chronic and degenerative process that causes progressive neurologic and physical deterioration. Axonal thicknesses based on OCT measurements decrease over time because of physiologic aging. Therefore, the significant but mild correlation $(r=-0.427, P=0.01)$ between OCT measures and age was expected.

Our results agree with those of Inzelberg et $a l^{22}$ and Altintas et $a l^{23}$, and confirm the peripapillary RNFL loss observed in PD patients for measurements made with spectral-domain OCT systems. Imaging techniques such as OCT are noninvasive, inexpensive, and fast, and may be useful for neurologic follow-up of the disease by providing information about its rate of progression. Recent RNFL analyses demonstrated the ability of OCT to evaluate the central nervous system, and to provide biomarkers of progression and diagnosis in multiple sclerosis. ${ }^{28}$ This study contributes to this field by analyzing a range of parameters in PD patients using two next-generation OCT devices.

In conclusion, PD causes axonal damage in the RNFL along with retinal thinning that can be detected using
Fourier-domain OCT. Longitudinal studies with a larger sample are needed to corroborate our results and to evaluate the usefulness of RNFL measurements as biomarkers of PD progression.

\section{Summary}

What was known before

- Spectral-domain OCT has been used in only two previous studies.

- Macular thickness was the only parameter found to be significantly altered in PD patients compared with controls, but RNFL measurements were not analyzed.

What this study adds

- Ability of spectral-domain OCT to detect retinal nerve fiber atrophy in Parkinson were evaluated.

- Both devices were performed in order to interpret results in clinical practice.

\section{Conflict of interest}

The authors declare no conflict of interest.

\section{Acknowledgements}

We thank the Parkinson's Disease Association of Aragon (Asociación de Parkinson de Aragón) for their help and participation in this project. All subjects gave detailed consent to participate in this study, which was conducted in accordance with the guidelines established by the Ethics Committee of the Miguel Servet Hospital and based on the principles of the Declaration of Helsinki.

\section{References}

1 Park A, Stacy M. Non-motor symptoms in Parkinson's disease. J Neurol 2009; 256(Suppl 3): 293-298.

2 Braak H, Del Tredici K, Bratzke H, Hamm-Clement J, Sandmann-Keil D, Rüb U. Staging of the intracerebral inclusion body pathology associated with idiopathic Parkinson's disease (preclinical and clinical stages). J Neurol 2002; 249(Suppl 3): III/1-III/5.

3 Bodis-Wollner I. Retinopathy in Parkinson disease. I Neural Transm 2009; 116(11): 1493-1501.

4 Bodis-Wollner I, Yahr MD, Mylin LH, Thornton J. Dopaminergic deficiency and delayed visual evoked potentials in humans. Ann Neurol 1982; 11(5): 478-483.

5 Moschos MM, Tagaris G, Markopoulos I, Margetis I, Tsapakis S, Kanakis M et al. Morphologic changes and functional retinal impairment in patients with Parkinson disease without visual loss. Eur J Ophthalmol 2011; 21(1): 24-29.

6 Schuman JS, Pedut-Kloizman T, Hertzmark E, Hee MR, Wilkins JR, Coker JG et al. Reproducibility of nerve fibre layer thickness measurements using optical coherence tomography. Ophthalmology 1996; 103(11): 1889-1898. 
7 Palace J. Inflammation versus neurodegeneration: consequences for treatment. J Neurol Sci 2007; 259: 46-49.

8 Hauser SL, Oksenberg JR. The neurobiology of multiple sclerosis: genes, inflammation, and neurodegeneration. Neuron 2006; 52: 61-76.

9 Sá MJ. Physiopathology of symptoms and signs in multiple sclerosis. Arq Neuropsiquiatr 2012; 70(9): 733-740.

10 Garcia-Martin E, Pueyo V, Pinilla I, Ara JR, Martin J, Fernandez J. Fourier-domain OCT in multiple sclerosis patients: reproducibility and ability to detect retinal nerve fiber layer atrophy. Invest Ophthalmol Vis Sci 2011; 52(7): 4124-4131.

11 Hajee ME, March WF, Lazzaro DR, Wolintz AH, Shrier EM, Glazman $S$ et al. Inner retinal layer thinning in Parkinson disease. Arch Ophthalmol 2009; 127(6): 737-741; Erratum in: Arch Ophthalmol 2009;127(12): 1615.

12 Cubo E, Tedejo RP, Rodriguez Mendez V, López Peña MJ, Trejo Gabriel Y, Galán JM. Retina thickness in Parkinson's disease and essential tremor. Movement Disorders 2010; 25: 2461-2477.

13 Aaker GD, Myung JS, Ehrlich JR, Mohammed M, Henchcliffe C, Kiss S. Detection of retinal changes in Parkinson's disease with spectral-domain optical coherence tomography. Clin Ophthalmol 2010; 4: 1427-1432.

14 Bodis-Wollner I, Spund B, Liu T, Shrier E, Selesnick I, Glazman $S$ et al. Remodeling of the fovea in Parkinson disease. Invest Ophthalmol Vis Sci 2011; 52(E-Abstract): 6660.

15 Rohani M, Langroodi AS, Ghourchian S, Falavarjani KG, Soudi R, Shahidi G. Retinal nerve changes in patients with tremor dominant and akinetic rigid Parkinson's disease. Neurol Sci 2012; e-pub ahead of print 3 June 2012; doi:10.1007/s10072-012-1125-7.

16 Hughes AJ, Daniel SE, Kilford L, Lees AJ. Accuracy of clinical diagnosis of idiopathic Parkinson's disease. A clinico-pathological study of 100 cases. Neurodegener Dis 2010; 7(5): 284-290.

17 Reichmann H. Clinical criteria for the diagnosis of Parkinson's disease. Neurodegenerative Dis 2010; 7: 284-290.

18 De Lau LM, Breteler MM. Epidemiology of Parkinson's disease. Lancet Neurol 2006; 5: 525-535.

19 Hoehn MM, Yahr MD. Parkinsonism: onset, progression and mortality. Neurology 1967; 17(5): 427-442.

20 Gupta PK, Asrani S, Freedman SF, El-Dairi M, Bhatti MT. Differentiating glaucomatous from non-glaucomatous optic nerve cupping by optical coherence tomography. Open Neurol J 2011; 5: 1-7.
21 Early Treatment Diabetic Retinopathy Study Research Group. Photocoagulation for diabetic macular edema. Early Treatment Diabetic Retinopathy Study Report No. 1 1985; 103: 1796-1806.

22 Inzelberg R, Ramirez JA, Nisipeanu P, Ophir A. Retinal nerve fiber layer thinning in Parkinson disease. Vision Res 2004; 44(24): 2793-2797.

23 Altintaş O, Işeri P, Ozkan B, Cağlar Y. Correlation between retinal morphological and functional findings and clinical severity in Parkinson's disease. Doc Ophthalmol 2008; 116(2): 137-146.

24 La Morgia C, Barboni P, Rizzo G, Carbonelli M, Savini G, Scaglione $\mathrm{C}$ et al. Loss of temporal retinal nerve fibers in Parkinson disease: a mitochondrial pattern? Eur J Neurol 2013; 20: 198-201.

25 Archibald NK, Clarke MP, Mosimann UP, Burn DJ. Retinal thickness in Parkinson's disease. Parkinsonism Relat Disord 2011; 17(6): 431-436.

26 Sung KR, Wollstein G, Bilonick RA, Townsend KA, Ishikawa $\mathrm{H}$, Kagemann $\mathrm{L}$ et al. Effects of age on optical coherence tomography measurements of healthy retinal nerve fiber layer, macula, and optic nerve head. Ophthalmology 2009; 116(6): 1119-1124.

27 Garcia-Martin E, Pueyo V, Ara J, Almarcegui C, Martin J, Pablo L et al. Effect of optic neuritis on progressive axonal damage in multiple sclerosis patients. Mult Scler 2011; 17(7): 830-837.

28 Garcia-Martin E, Pablo LE, Herrero R, Satue M, Polo V, Larrosa JM et al. Diagnostic ability of a linear discriminant function for Spectral domain optical coherence tomography in multiple sclerosis patients. Ophthalmology 2012; 119(8): 1705-1711.

29 Kesler A, Vakhapova V, Korczyn AD, Naftaliev E, Neudorfer M. Retinal thickness in patients with mild cognitive impairment and Alzheimer's disease. Clin Neurol Neurosurg 2011; 113(7): 523-526.

30 Shrier EM, Adam CR, Spund B, Glazman S, Bodis-Wollner I. Interocular asymmetry of foveal thickness in Parkinson disease. J Ophthalmol 2012; 2012: 728457.

31 Nakatani Y, Higashide T, Ohkubo S, Takeda H, Sugiyama K. Evaluation of macular thickness and peripapillary retinal nerve fiber layer thickness for detection of early glaucoma using spectral domain optical coherence tomography. J Glaucoma 2011; 20(4): 252-259. 